THE BENJAMIN LEVICH INSTITUTE

FOR PHYSICO-CHEMICAL HYDRODYNAMICS

THE CITY COLLEGE OF THE CITY UNIVERSITY OF NEW YORK

\author{
Progress Report on Activities under the U.S. Department of Energy \\ Grant \# DEF-G02-88ER13822
}

\title{
STUDIES IN PREMIXED COMBUSTION
}

\author{
Gregory I. Sivashinsky - Principal Investigator \\ November 1, 1990 - October 31, 1992
}

During the period under review a significant progress has been made along the principal lines of the project. A briet $z$,ccount of the results obtained follows.

\section{DISCLAIMER}

\begin{abstract}
This report was prepared as an account of work sponsored by an agency of the United States Goversment. Neither the United States Government not any agency thereof, nor any of their employees, makes any warranty, express or implied, or assumes any legal liability or responsibility for the wecuracy, completeness, or usefulness of any information, appazatus, product, or process disclosed, or represents that its use would not infringe privately owned rights. Refer. erice herein to any specific commercial product, process, or service by trade name, trademark, inanufacturer, or otherwise does not necessarily constitute or imply its endorsernent, recom. mendation, or favoring by the United Stateid Government or any agency thereof. The vicws and opinions of authors expressed herein do not necessarily state or reflect thase of the United States Government or any agency thereof.
\end{abstract}

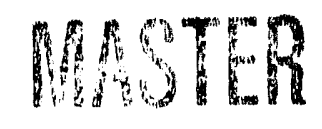




\section{THEORY OF TURBULEN'T FLAME PROPAGATION}

1.1. Pockets in Premixed Combustion and Combustinn Rate. It is generally accepted that the principal reason for the flame speed enhancement by large scale turbulence is the increase in the total flame interface. Moreover, as has been recently shown by Kerstein, Ashurst and Williams (Phys. Rev. A37, 1988, 2738), this is true irrespective of whether the flame is connected or includes pockets of unburned gas. On the other hand, it is clear (at least within the framework of the passive front formulation) that the pockets of unburned gas, being isolated from the leading front, may not infuence its propagation. The surface area of the pockets thus seems to be unable to contribute to the overall turbulent speed. This, however, contradicts the conventional point of view on the phenomenon. The present study is intended to resolve this apparent paradox of the flame-flow interaction dynamics.

[1] G. Joulin and G.I. Sivashinsky, Combustion Science and Technology (1991) 77, NN4-6 p.329.

\subsection{On Flame Propagation through Periodic Flow Fields. Flame propagatiors} through a periodic flow-field, for all its conceptional simplicity, is still a racher poorly studies aspect of flame-flow interaction. As has been recently realized, the solution of this problem is of fundamental interest for understanding the character of flame propagation in a turbulent flow-field - one of the central problems of combustion fluid dynamics. Considering the turbulent flow as a suitably arranged superposition of one-scale flows, the speed of the turbulent flame may be regarded as a renormalization of the effective flame speed induced by one-scale flow-fields. As shown by Clavin \& Williams (J. Fluid Mech. 90, 1979, 589 ) the effective (turbulent) speed of the flame propagating through a large scale and low intensity isotrupic flow-field has a global (integral) dependence on the underlying flow-field. It transpires, however, that this is true only if the level of flow-field fluctuations is low enough. As we show in this paper, at high amplitudes of the velocity spatial variations, due to the hyperbolic nature of the flame front dynamics, 
the effective speed of the flame becomes sensitive to the local features of the flow-field. Simultaneously, the flame undergoes a transition from a smooth configuration to a cusped one and may even create pockets of unburned gas.

\section{[2] Wm. T. Ashurst and G.I. Sivashinsky, Combustion Science and} Technology (1991), vol.80, p.159.

\subsection{Renormalization of Markstein's Length in Premixed Flames Moving} Through the Turbulent Flow-Field. As is well known, normal speed $U_{F}$ of a weakly distorted laminar flame moving through a motionless gas depends on the local curvature of the flame front $R^{-1}$, according to the relation

$$
U_{F}=U_{0}\left(1-\ell_{M} R^{-1}\right)
$$

where $U_{0}$ is the speed of a planar laminar flame and $\ell_{M}$ is the characteristic (Markstein's) length controlled by the physico-chemical properties of the mixture. In the presence of the turbulent flow-field Eq.(1) undergoes modification, $R^{-1}$ being replaced by the socalled flame stretch $\mathcal{K}$, which reflects the combined effect of the local flame curvature and the strain imposed by the underlying flow. However, as we show in the present study, if the largest length-scale associated with the curved flame markedly exceeds the integral scale of the turbulent flow-field (assumed to be isotropic and homogeneous) the functional dependence expressed by Eq.(1) still holds provided $\ell_{M}$ is replaced by some effective Markstein's length, while $U_{0}$ and $U_{F}$ are regarded as speeds of 'planar' and 'curved' turbulent flames correspondingly.

\section{[3] G.I. Sivashinsky, in preparation.}

1.4. Flame Extinction by Periodic Flow Field. It has long been observed that for each gaseous corrbustible mixture there is a certain level of turbulence at which the speed of the premixed flame reaches its maximal value. A further increase in the intensity of turbulence leads to the drop of the flame speed and eventual extinction of the flame.

The turbulent flow field, as it is well known, is characterized by a wide range of spatio-ternporal scales involved. This constitutes the main difficulty of the corresponding mathematical prublems. It seems intuitivel r plausible, however, that the multiple - scale 
nature of the flow-field has little to do with the basic physics of the flame extinction which may well be described within the framework of a rather sirnple one-scale flameflow interaction scheme. Moreover, to capture the basic effect the flow field may be considered as time-independent, space-periodic and even unidirectional (Berestycki \& Sivashinsky, SIAM J. Appl. Math (1991) 51, 344).

In the present stidy, the direct numerical simulation of the phenomenon is undertaken. The preliminary results obtained show, that near the extinction threshold only certain portions of flame go out which, nevertheless, causes a marked drop in flame propagation speed. Further increase in the level of the flow-field fluctuations is expected to lead to a total extinction of the flame.

\section{[4] G. Kozlowsky and G.I. Sivashinsky, in preparation.}

These results are reported in the D.Sc. Thesis "An Interactive Programming Environment for Solving Partial Differential Equations Using Adaptive Grids (with application to flame-flow interaction problems)" by G. Kozlovsky, whose studies were supported by the grant.

In the absence of molecular diffusivity and assuming the velocity profile to be a stepwise periodic function, the original problem may be associated with the wave of gasless combustion moving through the pile of alternatingly sliding layers of solid fuel. Unlike the gaseous case such a system is quite feasible experimentally. The system seems also to be advantageous from the modeling point of view. It is well known that the main features of flame extinction in cooled ducts are successfully described within the framework of a one-dimensional formulation, where heat exchange with the environment is perceived as a volumetric heat loss. This approach proves effective in the description of gasless combustion of the above layered system as well. The pertinent one-dimensional formulation is clearly much more treatable both analytically and numerically.

The preliminary numerical simulations show that at slow sliding, combustion waves of both layers move in tandem, whose velocity is somewhat lower than in the absence of sliding. However, if the sliding speed exceeds a certain critical value for overall picture undergoes a considerable change.

The flame is, as it were, torn apart by the flow. In the layers moving in the direction 
of the flame spreading combustion quenches, but survives in oppositely moving layers. Thus, the periodic flow-field indeed promotes extinction - at least partial.

\section{[5] I. Brailovsky and G.I. Sivashinsky, in preparation.}

\section{PATTERN FORMATION IN PREMIXED FLAMES AND RELATED PROBLEMS}

\subsection{On Slot-Burner Polyhedral Flames. Bunsen burner polyhedral flames are} one of the most graphic manifestations of cellular instability in premixed gas combustion. A vast experimental data is accumulated on this both striking and elegant phenomenon. While the basic mechanisms underlying the cellular flame instability are believed to be understood reasonably well, the progress in the actual description of the three-dimensional polyhedral flames remains rather limited. There are still many questions concerning the sensitivity of the polyhedral instability to the composition of the mixture and to the burner rim aerodynamics. As a first step, clearly, it is important to examine the potential of the intrinsic front dynamics when the upstream flow-field is regarded as unidirectional and uniform, while the role of the burner rim is reduced merely to keeping the flame above the prescribed border. The present study provides numerical simulations of slot-burner premixed flames within the framework of a weakly nonlinear model for flame interfact dynamics. In a qualitative agreement with experimental observations, the results cbtained show that the occurrence of the polyhedrals is largely controlled by the conditions at the burner rim. On the other hand, the model does not seem to be capable of capturing the well known traveling mode of polyhedral instability and requires an appropriate modification.

[6] S.M. Gutman, R.L. Axelbaum, C.K. Law and G.I. Sivashinsky, Cornbustion Science and Technology (1992), submitted for publication.

We are currently working on an extension of the above results of the situation of the circular burner rim. 


\subsection{Negative Burning Speed in Oscillatory Premixed Gas Combustion.}

As is well known, planar waves of prernixed gas combustion may spontaneously assume as oscillatory mode of propagation, provided the molecular diffusivity of the limiting reactant sufficiently exceeds the thermal diffusivity of the mixture. The phenomenon way be successfully described within the framework of the pertinent reaction-diffusion model (Matkowsky \& Sivashinsky, SIAM J. Appl. Math. (1979) 37, 687). Recently Frankel (Phys. Letters A, (1989) 140,405) employing a rather unconconventional averaging approach, succeeded to reduce the reaction-diffusion problem to a second order O.D.E., which admits to a stable limit cycle. One of the fascinating implementations of Frankel's theory is its prediction of the backward motion of the flame occurring within certain time intervals, if the system is shifted sufficiently deeply into the instability domain. The negative burning speed does not contradict the physics of premixed gas combustion, provided the diffusive term of the total heat flux exceeds its convective counterpart. Nevertheless, due to the essentially extrapolative nature of Frankel's model, one cannot be certain whether the negative speed effect he observed is indeed peculiar to the original reaction-diffusion system, or whether it is merely an artifact induced by the approximation. In the present study a direct numerical simulation of the oscillatory flame motion is carried out. The results obtained show that inversion of the flame speed is indeed possible. It is also observed that sufficiently far from the stability threshold the oscillations may undergo period doubling.

[7] I. Brailovsky and G.I. Sivashinsky, Combustion Science and Technology (1992), accepted for publication.

\subsection{Structure of the Bunsen Flame. A Numerical Study.}

It is well known that the structure of the Bunsen flame is strongly sensitive to the deficient reactant's Lewis number $(L e)$. If thermal diffusivity of the premixture sufficiently exceeds the molecular diffusivity ( $L e>1$ ), the fame assumes a continuous luminous conical shape. However, in the opposite case, when molecular diffusivity is high enough $(L e<1)$, the flame appears "open" near its tip (e.g. Lewis \& von Elbe, 1961).

The analytical description based on high activation energy asymptotics succeeded 
in capturing many basic features of the phenomenon (Sivashinsky, J.Chem. Phys. 1975, 62, 638). At $\left(L_{e}<1\right)$, the temperature, reaction rate and flame speed were found to suffer a dramatic drop as the reaction zone approaches the tip. In order to maintain the flame at the tip its speed should be equal to that of the on-coming gas flow. In light of the observed tendencies it was plausible to expect that near the tip the flame simply goes out. Further analytical studies of the problem seemed to support this assertion.

For all that, the previous theories being asymptotic in nature were, strictly speal:ing, not valid at the extremity of the tip. To obtain a comprehensive picture it is therefore very instructive to undertake a direct numerical simulation of the phenomenon.

A detailed numerical study of the Bunsen burner flame is presented. It is shown that for the Lewis numbers exceeding unity the reaction rate and the flame speed gradually increase towards the flame tip. For small Lewis numbers the picture is quite different. The reaction rate drops near the tip. In spite of this the flame survives and, moreover, manages to to consume all the fuel supplied to the reaction zone. There is no leakage of the fuel through the front. The flame speed varies nonmonotonously along the front from gradual reduction to steep increase near the tip.

\section{[8] G. Kozlovsky and G.I. Sivashinsky, Combustion Science and Technology (1991), submitted for publication.}

\subsection{Chaotic Dynamics in Solid Fuel Combustion.}

Thermal feedback in exothermic chemical reactions can lead to a rich variety of nonrelaxational dynamical behavior. One of the simplest systems of this kind is a well known gasless combustion where solid fuei is transformed directly into a solid product. Apart from its relevance to high temperature synthesis technology which enjoys much attention recently, this process is also of interest as an example of a simple determiristic system capable of chatoc spatio-temporal dynamics.

In the present paper, the gasless combustion is studied within the well known freeboundary formulation. In qualitative ageement with recent numerical results established for the model employing the distributed reaction rate, it is shown that one-dimensional flame propagation may undergo highly irregular relaxational oscillations provided the 
bifurcation parameter (Zeldovich number) is high enough. Increasing the bifurcation parameter leads to an enhancement of the level of fluctuation and a reduction of the average flame speed.

\section{[9] I. Brailovsky and G.I. Sivashinsky, Physica D: Nonlinear Phenomena (1992), submitted for publication.}

Transition to chaos is preceded by a succession of period doublings where one may identify at least four consecutive bifurcations.

[10] M. Frankel, V. Foytburd and G. Sivashinsky, SIAM J. on Applied Math. (1992), submitted for publication.

Apart from the numerical simulations some features of oscillatory combustion may also be described analytically. Employing a new high order slowly-varying-flame formalism (cf. Sivashinsky, Acta Astronautica (1976) 3, 889) the pertinent reaction-diffusion problem is reduced to a second order O.D.E., admitting to a stable limit cycle. Analytical solutions of this asymptotic equation are compared with the results of direct numerical simulations of the original reaction-diffusion model.

\section{[11] A. Efet and G. Sivashinsky, in preparation.}

\subsection{Instabilities and Pattern Formation in Large-Scale Premixed Flames Propagating Between Closely Spaced Horizontal Plates.}

The planar flame is clearly the most simple configuration of combustion wave. While such flames are quite feasible in the laboratory, it is also known that under certain physico-chemical conditions flames spontaneously assume a more complex two - or three dimensional structure. There are two basic reasons for spontaneous flame instability: (i) low Lewis number (high mobility) of the deficient reactant and (ii) thermal expansion of the burned gas. The first mode of flame instability, which manifests itself in the appearance of the small-scale cellular structure is relatively easily produced in small scale systerns under normail laboratory conditions. On the other hand, our understanding of the hydrodynamic (thermal expansion) mode of flame instability (ii) is much less satisfactory. In order to carry out a systematic study of a developed mode of hydrodynamic instability, the aspect ratio of the pertinent premixed system should be sufficiently large. 
This invariably encounters substantial technical difficulties both in the attempts to conduct the systematic experimental study of the system and in its numerical simulation. In the present work we propose a new approach to the study of hydrodynamic flame instability based on lowering the effective dimensionality of the systern and at the same time without reducing its aspect ratio, thereby, hopefully preserving most of the basic features of the large-scale flame dynamics. Specifically, we consider the premixed flame freely evolving between two closely spaced horizontal plates - a gaseous analogue to a Hele-Shaw planar cell.

The proximity of the plates, however, induce such side effects as heat losses and viscous friction, which in turn markedly effect the dynamics of the flame and its stability limits.

[12] G. Joulin and G.I. Sivashinsky, Combustion Science and Technology, submitted for publication.

\subsection{On the Linear Hydrodynamic Stability and Response of Premixed Flames in Stagnation-Point Flow.}

Using potential models as computational tools, the linear stability of strained premixed flames held in 2-dimensional stagnation-point flows are studied. Three configurations, in which fresh gases respectively impinge: (a) burned ones, (b) a wall, (c) another jet of fresh mixture are considered. It is shown that:

- if intense enough, flow divergence can stabilize disturbances with wavevector normal to directions of strain;

- any stretch always stabilizes those wrinkles with wavevectors parallel to direction of positive strain;

- the least stable modes of wrinkling are antisymmetric disturbances of twin-fronts (cace, c) and configuration (b) is the most stable one.

Lastly, we study the linear response of axisymmetrically stretched flames (which are always linearly stable) to incorning random velocity fluctuations. Ensemble-averaged amplitude of wrinkling are theoretically estimated and a statistical tendency for twin fronts to mutually avoid each other is suggested. 
[13] G. Joulin and G.I. Sivashinsky, 24th Symposium (Int) on Combustion, Sydney, accepted.

\subsection{On Nonlinear Saturation in Premixed Flames Propagating Through the Shear Flow.}

The basic mechanism providing nonlinear saturation of growing disturbances in intrinsically unstable freely propagating flames, has a purely geometrical nature stemming from Huygens principle. In flames propagating through hydrodynamic fields new possibilities for nonlinear saturation may arise which may be comparable, or even dominate over Huygens stabilization. Such a situation occurs, for example, in shear flows. The flame evolution equation accounting for this mode of flame-flow interaction acquires a new nonlinear term quite similar to that appearing in the Boussinesq equation.

In intrinsically unstable systems this term is known to provide the nonlinear saturation of growing disturbances. At a very strong shear, the Boussinesq term overpowers the Huygens nonlinearity, markedly reducing the level of the interface fluctuations and thereby the speed of flame propagation.

[14] Y. Nikolaevsky and G.I. Sivashinsky, Combustion Science and Technology, submitted for publication.

\subsection{Quasi-Equilibrium in Upward Propagation Flames.}

Thermal expansion of the gas accompanying flame propagation makes the latter sensitive to external acceleration. In upward propagating flames, the cold (denser) mixture is superposed over the hot (less dense) combustion products. For this reason, the plane flame front separating the cold and hot gases is subject in this case to the classical effect of Rayleigh-Taylor instability. As a result, the flame front becomes convex towards the cold gas.

As is known from many experimental observations, upward propagating flames often assume a characteristic paraboloidal shape with the tip of the paraboloid located somewhere near the channel's centerline. Flames where the tip slides along the channel's wall has also been observed, however, this type of flame configuration somehow received less attention. Upward flame propagation, thus, may occur through different but seem- 
ingly stable geornetrical realizations. The present study is attempted to gain a better understanding of the pertinent nonlinear phenomenology, which transpires to be rather interesting.

It is argued that the parabolic fronts occurring in upward propagating flames may actually be merely quasi-equilibrium transient states which eventually collapse to a stable configuration in which the inclined flame as it were spreads along the wall of the channel.

[15] A.B. Mikishev and G.I. Sivashinsky, Physics Letters A, submitted for publication.

\subsection{Geometrically Invariant Theory of Cellular Flames.}

For premixed combustion to be actually maintained in the form of a steady planar reaction wave, its structure must be stable under small disturbances. Many flames are known to be planar under normal laboratory conditions. There is, however, also a class of flames that prefer a characteristic two- or three-dimensional cellular structure emerging as a result of a planar flame instability. Cellular flames have enjoyed much attention in recent years as an example of a relatively simple pattern forming system capable of random type behaviour.

Previous theoretical activity was focused primarily on the dynamics of the quasiplanar cellular flames occurring near the stability threshold. For all their rich phenomenology quasi-planar flames, however, do not cover such important systems as freely expanding spherical flames where the interfacial stretch may markedly reduce the limits and character of the instability.

A rational extension of the quasi-planar theory to describe flames of arbitrary geometrical configurations is the principal object of the present study.

Apart from a derivation of the tractable coordinate-invariant evolution equations,

the work also involves the large-scale numericai simulations which yield quite 'f 'few challenging technical problems yet to be resolved.

[16] L.V. Filyand and G.I. Sivashinsky, in preparation. 


\subsection{On Stabilization of Inverted Flames.}

Premixed inverted flames stabilized in' the wake of a gas flow past a cylindrical rod are studied. The limit of a nearly planar flame when the velocity of the oncoming flow is close to the normal flame speed is considered. The pertinent lame interface evolution equation describing the mechanism of flame attachment is derived and studied both analytically and numerically. It is shown that the flame stabilization is essentially a global phenomenon which cannot be adequately captured by local analysis. The principal mechanism of flame stabilization thus transpires to be of a basically kinematic nature where transport effects play merely a subordinate role. It is also shown that to hold the detached flame, the diameter of the rod should exceed a certain critical value comparable to the Markstein length of the system.

\section{[17] A.B. Mikishev and G.I. Sivashinsky, Physics of Fluids, submitted for publication.}

\subsection{Flame Propagation in a Horizontal Channel.}

When a gaseous mixture is burning in a horizontal channel, the flame front assumes a unique shape, which is not symmetric with respect to the channel axis. The upper part of the front projects forward in the direction of flame propagation. The physical reason for this shape is readily identified as follows. The combustion zone separates the fresh mixture, with its high density, from the light combustion products. The heavier gas always tends to extend along the lower part of the vessel, with the higher gas occupying the upper part. The first attempt to develop a mathematical description of the flame shape formed under these conditions was proposed in Rakib \& Sivashinsky (Comb. Sci. Tech. 1988, 24, 247-60), where a weak thermal expansion approximation was employed. The theory provides quite a satisfactory picture of the flame interface. However, the pertinent formula for the flame speed yields a rather poor extrapolation to the important region of wide channels where, according to the experimental data, the flame moves as a large gas bubble spreading in a tube filled with liquid.

In the present work this system is being studied employing the appropriately adjusted techniques of the conform mapping theory which shows much promise here. 
[18] Y. Shtemler and G. Sivashinsky, in preparation.

\section{PATTERN FORMATION IN EXTENDED SYSTEMS}

This section reports further progress on some issues related to our previous research project, "Topics in Physicom Chemical Hydrodynamics" under the same DOE Grant \# DEF-G02-88ER13821.

\subsection{On Negative Eddy Viscosity under Conditions of Isotropy.}

As has been recently realized there is quite an appreciable class of extended dissipative systerns where the effective transport coefficients under certain conditions may becorne negative, thereby promoting a spontaneous formation of large-scale structures. The cellular flames occurring in combustion of rich hydrocarbon-air gaseous premixtures is a typical example of such a situation. The flame splits up into large cells while the effective diffusivity of the pertinent pattern forming dynamics appears to be negative. It transpires that quite a similar phenomenology may emerge in the classical dynamics of viscous fluids, many formal fractures of which appear to be searly identical to those of the reaction-diffusion systems.

This study considers a large-scale flow freely evolving through a periodic array of oscillating triangular eddies. The effective eddy viscosity induced by the system appears to be isotropic, i.e., insensitive to the spatial orientation of the underlying periodic flowfield. In contrast to the previously studied situation with time-independent periodic flow, the eddy viscosity generated by rapidly oscillating eddies may be negative, thereby reducing the total (molecular and eddy) viscosity of the systern. At sufficiently high Reynolds numbers. even the total viscosity may assume negative values, thus initiating the inverse energy cascade and inducing occurrence of large-scale flow-fields.

[19] G.I. Sivashinsky and A.L. Frenkel, Physics of Fluids, to appear. I'

\subsection{Hexagonal Structure of Large-Scale Marangoni Convection.}

Another striking example of behavioural similarity between cellular flaroes and pattern forming hydrodynamical systems is provided by thermo-capillary (Marangoni) instability in thin liquid layers. In both systems the secondary structures emerging near 
the pertinent stability threshold assume the form of a hexagonal pattern. As we show in the present study, despite the completely different physical nature of the systems, the nonlinear dynamics governing formation of cellular flames and Marangoni convection (with a certain range of parameters) is nearly identical.

[20] L. Shtilman and G.I. Sivashinsky, Physica D. Nonlinear phenomena, (1991) $52,477-488$.

\subsection{Effects of Viscosity in the Shear-Flow Interfacial Instabilities.}

The undirectional shear flows involving interfaces are the classical example of pattern forming extended systems capable of random-type behaviour. Until now, all analytical studies of the pertinent dynamics regarded the appropriately nondimensionalized surface tenion as a large parameter. The advantage of this limit is that it brings in the natural separation of scales and thus allows to extract an explicit equation for the interface evolution. The iypical size of the wavy pattern emerging on the interface thus appears to be invariably controlled by the surface tension. In the systems involving very thin layers such an outcome correlates well with the observations. However, in many extended shearflow systems the surface tension is not large and therefore cannot control the pattern wavalength.

As we intend to show in this study, in the shear flow systems of finite surface tension the latter merely ensures a dissipation of the short-wave disturbances. However, the pattern wavelength is controlled by the viscosity. As a result the pertinent interface evolution equation assumes a nonlocal form, resembling that appearing in the theory of hydrodynamic flane instability.

[21] A. Oron and G.I. Sivashinsky, in preparation. 

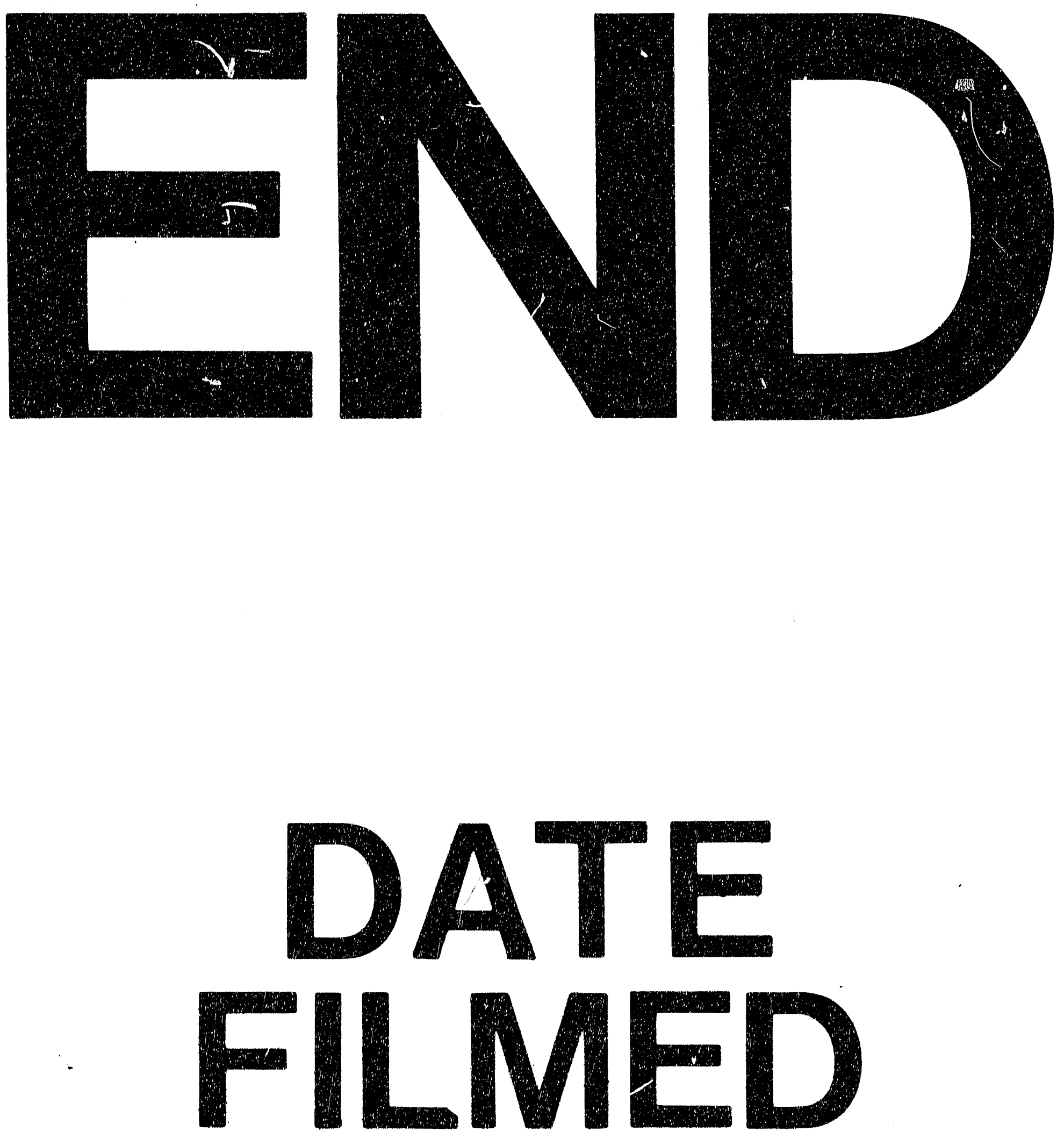

1

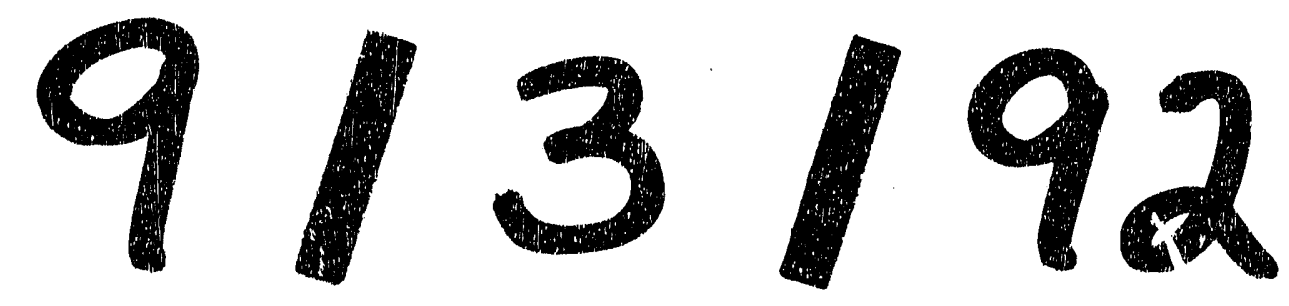


